\title{
A note on the prime numbers of the forms
}

$$
N=(6 a+1) 2^{2 n-1}-1 \text { and } M=(6 a-1) 2^{2 n}-1
$$

By Hans Rreseu

The purpose of this paper is to give a direct proof of the following two criteria for the primality of the numbers of the above forms. The theorems are, except for a slight modification of the conditions, known by LeHMER [1] and BRewer [2] who proved a more general theorem of this kind. Then we take up to discussion if the third inequality in the theorems could be removed, and show that this is not the case. Finally we give a numerical example and a table of some primes of the discussed forms.

Theorem 1. Let $a$ and $n$ be integers satisfying

$$
a \geqq 0, n \geqq 2,2^{2 n-1}>a .
$$

Let a sequence $u_{i}$ of integers be defined by the recurrence relation

with

$$
u_{i+1}=u_{i}^{2}-2 \quad(i=1,2,3, \ldots)
$$

$$
u_{1}=(2+\sqrt{3})^{6 a+1}+(2-\sqrt{3})^{6 a+1} \text {. }
$$

Then a necessary and sufficient condition that the number $N=(6 a+1) 2^{2 n-1}-1$ should be a prime is that

$$
u_{2 n-2} \equiv 0(\bmod N)
$$

Theorem 2. Let $a$ and $n$ be integers satisfying

$$
a \geqq 1, n \geqq 2,2^{2 n}-1 \geqq a \text {. }
$$

Let a sequence $v_{i}$ of integers be defined by the recurrence relation

with

$$
v_{i+1}=v_{i}^{2}-2 \quad(i=1,2,3, \ldots),
$$

$$
v_{1}=(2+\sqrt{3})^{6 a-1}+(2-\sqrt{3})^{6 a-1} \text {. }
$$

Then a necessary and sufficient condition that the number $M=(6 a-1) 2^{2 n}-1$ should be a prime is that

$$
v_{2 n-1} \equiv 0(\bmod M) \text {. }
$$




\section{H. RIESEL, Prime numbers of two forms}

Preliminary remarks. The proofs of the two theorems will follow the same lines and are very similar. Here we give the full details of the proof only for Theorem 1, and sketch the proof of Theorem 2. The first part of the proof follows a proof by WESTERN [3] for the special case $a=0$ in Theorem 1 .

The proof is based on some results from the theory of the quadratic number field $K(\sqrt{3})$, where $K$ is the field of rational numbers. The field $K(\sqrt{3})$ has the following well-known properties:

The integers $x$ in the field are of the form $x=a+b \sqrt{3}$, where $a$ and $b$ are rational integers. The units are $\pm(2+\sqrt{3})^{n}, n$ a rational integer. The conjugated number $\bar{x}$ of $x=a+b \sqrt{3}$ is $\bar{x}=a-b \sqrt{3}$. The norm $N(x)$ of $x$ is defined by $N(x)=x \bar{x}=a^{2}-3 b^{2}$.

There are two kinds of primes in the field (excepting the factors of 2 and 3). Firstly, all rational primes $q$ of one of the forms $q=12 s \pm 5$. Secondly, all primes $\pi=a+b \sqrt{3}$, whose norm $\pi \bar{\pi}=a^{2}-3 b^{2}$ equals a rational prime $p$ of one of the forms $p=12 s \pm 1$.

We need the following analogue of Fermat's theorem: Let $q$ be a prime of one of the forms $q=12 s \pm 5$. Then, if $x$ is an integer in the field, we have

$$
x^{q} \equiv \bar{x}(\bmod q) .
$$

Proof: Let $x=a+b \sqrt{3}$. Then we have

$$
x^{q}=(a+b \sqrt{3})^{Q} \equiv a^{q}+b^{a}(\sqrt{3})^{q} \equiv a+b \cdot(3)^{\frac{q-1}{2}} \sqrt{3} \equiv a-b \sqrt{3}=\bar{x}(\bmod q)
$$

by the binomial theorem, Fermat's theorem for rational integers and since

$$
3^{\frac{q-1}{2}} \equiv\left(\frac{3}{q}\right)=-1(\bmod q)
$$

$\left(\left(\frac{3}{q}\right)\right.$ is Legendre's symbol, and $\left(\frac{3}{q}\right)=-1$ if $\left.q=12 s \pm 5\right)$.

We also require the following lemma: Let $x$ be an integer of the field $K(\sqrt{3})$. Let $d$ (if it exists) be the smallest positive exponent for which the congruence

$$
x^{d} \equiv-1(\bmod q) \quad(q \text { prime })
$$

holds. Then, if $\omega$ is any exponent for which one of the congruences

$$
x^{\omega} \equiv-1(\bmod q) \text { or } x^{\omega} \equiv 1(\bmod q)
$$

holds, $\omega$ is an odd or an even multiple of $d$, respectively.

Since the corresponding lemma for the rational integers is well known and since the proof in the field $K(\sqrt{3})$ is the same, we omit the proof.

Proof of Theorem 1. We find $N=(6 a+1) 2^{2 n-1}-1 \equiv-1(\bmod 8)$ (since $n \geqq 2)$ and $N=(6 a+1) 4^{n-1} \cdot 2-1 \equiv(6 a+1) \cdot 4 \cdot 2-1 \equiv 7(\bmod 12)$. 
First, we prove the necessity of condition (2). Suppose $N$ is a prime. Then Legendre's symbols $\left(\frac{-1}{N}\right),\left(\frac{2}{N}\right)$ and $\left(\frac{-2}{N}\right)$ have the values

and

$$
\left(\frac{-1}{N}\right)=-1,\left(\frac{2}{N}\right)=+1,\left(\frac{-2}{N}\right)=-1
$$

$$
\left(\frac{-2}{N}\right) \equiv(-2)^{\frac{N-1}{2}}=(1+\sqrt{3})^{\frac{N-1}{2}}(1-\sqrt{3})^{\frac{N-1}{2}} \equiv-1(\bmod N)
$$

or

or

$$
\left(\frac{1+\sqrt{3}}{1-\sqrt{3}}\right)^{\frac{N-1}{2}}(1-\sqrt{3})^{N-1} \equiv-1(\bmod N)
$$

$$
\left(\frac{1+\sqrt{3}}{1-\sqrt{3}}\right)^{\frac{N-1}{2}} \frac{2}{1-\sqrt{3}}(1-\sqrt{3})^{N} \equiv-2(\bmod N) .
$$

Now $(1-\sqrt{3})^{N} \equiv 1+\sqrt{3}(\bmod N)$, according to $(3)$, and cancelling the factor 2 , we find

$$
\left(\frac{1+\sqrt{3}}{1-\sqrt{3}}\right)^{\frac{N+1}{2}} \equiv-1(\bmod N) \text {. }
$$

Since $\frac{1+\sqrt{3}}{1-\sqrt{3}}=\frac{(1+\sqrt{3})^{2}}{-2}=-(2+\sqrt{3})$, we find

or since $(-1)^{\frac{N+1}{2}}=1$,

$$
(-1)^{\frac{N+1}{2}}(2+\sqrt{3})^{\frac{N+1}{2}} \equiv-1(\bmod N)
$$

$$
(2+\sqrt{3})^{\frac{N+1}{2}} \equiv-1(\bmod N)
$$

Adding 1 and multiplying by $(2-\sqrt{3})^{\frac{N+1}{4}}$, we see that congruence (6) implies

$$
(2+\sqrt{3})^{\frac{N+1}{4}}+(2-\sqrt{3})^{\frac{N+1}{4}} \equiv 0(\bmod N) \text {. }
$$

This, however, is the same as condition (2), since

$$
\begin{aligned}
& u_{1}=(2+\sqrt{3})^{6 a+1}+(2-\sqrt{3})^{6 a+1} \text {, } \\
& u_{2}=u_{1}^{2}-2=(2+\sqrt{3})^{(6 a+1) 2}+(2-\sqrt{3})^{(6 a+1) 2} \text {, } \\
& \text {............... } \\
& u_{2 n-2}=u_{2 n-3}^{2}-2=(2+\sqrt{3})^{(6 a+1) 2^{2 n-3}}+(2-\sqrt{3})^{(6 a+1) 2^{2 n-3}} \\
& =(2+\sqrt{3})^{\frac{N+1}{4}}+(2-\sqrt{3})^{\frac{N+1}{4}} .
\end{aligned}
$$

This shows the necessity of condition (2), if $N$ is a prime. 


\section{H. RIESEL, Prime numbers of two forms}

We now proceed to prove the sufficiency of the conditions given in Theorem 1. Suppose that $u_{2 n-2} \equiv 0(\bmod N)$, or, which is the same thing, that congruence (7) holds. By multiplying this congruence by $(2+\sqrt{3})^{(N+1) / 4}$ we obtain congruence (6). Now, since $N$ has the form $12 s+7$, and since the product of primes of the forms $12 \mathrm{~s} \pm 1$ yield a number of the same form, $N$ must have a prime factor $q$ of one of the forms $12 s \pm 5$. Since congruence (6) holds for the modulus $N$, it holds, a fortiori, for any modulus dividing $N$, and we obtain

$$
(2+\sqrt{3})^{\frac{N+1}{2}} \equiv-1(\bmod q) .
$$

The analogue of Fermat's theorem gives us

or

$$
(2+\sqrt{3})^{Q} \equiv 2-\sqrt{3}(\bmod q),
$$

$$
(2+\sqrt{3})^{q+1} \equiv 1(\bmod q) \text {. }
$$

Suppose now that $d$ is the smallest positive exponent for which

$$
(2+\sqrt{3})^{d}=-1(\bmod q) \text {. }
$$

Using the lemma from the preliminary remarks, we conclude that

$$
\frac{N+1}{2}=(6 a+1) 2^{2 n-2}=k d \quad(k \text { an odd number })
$$

and that

$$
q+1=j d(j \text { an even number }) .
$$

Since $k$ is odd, $d$ must contain the factor $2^{2 n-2}$, and since $j$ is even, $q+1$ contains the factor $2^{2 n-1}$. Hence we see that if $N$ were not prime we should find a factorisation of the form

$$
N=(6 a+1) 2^{2 n-1}-1=z \cdot q=z\left(m \cdot 2^{2 n-1}-1\right),
$$

with a $z>1$. If we consider this factorisation $\left(\bmod 2^{2 n-1}\right)$, we find that $z \equiv 1\left(\bmod 2^{2 n-1}\right)$, and that $N$ must have the form

$$
N=(6 a+1) 2^{2 n-1}-1=\left(l \cdot 2^{2 n-1}+1\right)\left(m \cdot 2^{2 n-1}-1\right) .
$$

The case $l=0, m=6 a+1$ corresponds to the trivial decomposition $N=1 \cdot N$.

Decomposition (8) implies

$$
6 a+1=l m \cdot 2^{2 n-1}+m-l=f(l, m) \text { say. }
$$

Considering $(9)(\bmod 2)$ and $(\bmod 3)$, we obtain

and

$$
1 \equiv m-l(\bmod 2)
$$

$$
\mathrm{l}+l m \equiv m-l(\bmod 3) \text {. }
$$


From these congruences it follows that the values of $m$ and $l$ :

are impossible.

$$
\begin{aligned}
& m=1, l=1,2,3,4,5 \\
& m=2, l=1,2 \\
& m=3, l=1 \\
& m=4, l=1,2 \\
& m=5, l=1,2,3
\end{aligned}
$$

We shall now obtain the smallest possible value of the function $f(l, m)$. Since $f(l, m)$ is an increasing function of $l$ and of $m$, and since $l>m$ implies $f(l, m)<f(m, l)$, we find:

If $m=1, l$ must be $\geqq 6$, and

$$
f(l, 1) \geqq f(6,1)=6 \cdot 2^{2 n-1}-5 .
$$

In the same way we find:

and finally, if $m \geqq 6$

$$
\begin{aligned}
& f(l, 2) \geqq f(3,2)=6 \cdot 2^{2 n-1}-1, \\
& f(l, 3) \geqq f(2,3)=6 \cdot 2^{2 n-1}+1, \\
& f(l, 4) \geqq f(3,4)=12 \cdot 2^{2 n-1}+1, \\
& f(l, 5) \geqq f(4,5)=20 \cdot 2^{2 n-1}+1
\end{aligned}
$$

$$
f(l, m) \geqq f(l, 6) \geqq f(1,6)=6 \cdot 2^{2 n-1}+5 .
$$

Since the smallest of these expressions is $6 \cdot 2^{2 n-1}-5$, this shows that $f(l, m)$ for those values of $l$ and $m$ which are compatible with the congruences (10), satisfies

$$
f(l, m)=6 a+1 \geqq 6 \cdot 2^{2 n-1}-5
$$

Hence, if the opposite inequality

$$
6 a+1<6 \cdot 2^{2 n-1}-5
$$

holds, we conclude that the decomposition (8), with any $l>0$, is impossible. The last inequality reduces to

$$
a<2^{2 n-1}-1
$$

and so we have proved Theorem 1 .

For Theorem 2 we find that $M$ satisfies the same congruence relations $(\bmod 8)$ and $(\bmod 12)$ as $N$, and so we have the congruences (6) and (7) in exactly the same manner, but with $M$ instead of $N$ :

$$
(2+\sqrt{3})^{\frac{M+1}{4}}+(2-\sqrt{3})^{\frac{M+1}{4}} \equiv 0(\bmod M) .
$$




\section{H. RIESEL, Prime numbers of two forms}

Since $M=(6 a-1) 2^{2 n}-1$, we find that $\left(7^{\prime}\right)$ implies $v_{2 n-1} \equiv 0(\bmod M)$, if $v_{i+1}=v_{i}^{2}-2(i=1,2,3, \ldots)$, and

$$
v_{1}=(2+\sqrt{3})^{6 a-1}+(2-\sqrt{3})^{6 a-1} .
$$

The proof of the sufficiency leads in the same way as for Theorem 1 to the decomposition

and to

$$
M=(6 a-1) 2^{2 n}-1=\left(l \cdot 2^{2 n}+1\right)\left(m \cdot 2^{2 n}-1\right)
$$

$$
6 a-1=l m \cdot 2^{2 n}+m-l=f^{\prime}(l, m) \text { say, }
$$

and to the congruences

and

$$
1 \equiv l-m(\bmod 2)
$$

$$
1+l m \equiv l-m(\bmod 3) \text {. }
$$

The impossible values (11) are impossible in this case too, and the smallest value of the function $f^{\prime}(l, m)$ again occurs for $l=6, m=1$, and finally; the inequality

$$
6 a-1<6 \cdot 2^{2 n}-5
$$

which makes $\left(8^{\prime}\right)$ impossible with an $l>0$ leads to

that is

$$
a<2^{2 n}-\frac{2}{3},
$$

$$
a \leqq 2^{2 n}-1
$$

Remark 1. If, in Theorem 1, $a=0$, we have the following theorem: The number $N=2^{2 n-1}-1$ is a prime, if and only if $u_{2 n m 2} \equiv 0(\bmod N)$, where $u_{i+1}=u_{i}^{2}-2(i=1,2,3, \ldots)$ and $u_{1}=4$.

This is the well-known Lucas's theorem for the primality of Mersenne's numbers. See Western [3].

Remark 2. It is natural to ask whether Theorem 1 holds if the inequality

$$
2^{2 n-1}>a
$$

is not fulfilled, or whether this inequality is essential.1 We are going to prove that at least some kind of extra condition is needed, if $2^{2 n-1}>a$ is not fulfilled.

To prove this, let us in the case $n=2$ try to construct a composed number $N$ for which congruence (6) holds. Then everything in Theorem 1 will be satisfied except, of course, the inequality $2^{2 n-1}>a$.

Suppose that $N=(6 a+1) \cdot 8-1=48 a+7$ is the product of three different primes $q_{i}=48 n_{i}+7 \quad(i=1,2,3)$ of the same form as $N$. Since $7^{2} \equiv 1(\bmod 48)$

1 This problem does not seem to have been treated before. 
ARKIV För MATEMATIK. Bd 3 nr 20

and $7^{3} \equiv 7(\bmod 48)$, this is no contradiction. From the proof of the necessity of condition (2) in Theorem 1 it now follows that

$$
(2+\sqrt{3})^{\frac{a_{i}+1}{2}}=-1\left(\bmod q_{i}\right) \quad(i=1,2,3)
$$

A sufficient condition that

$$
(2+\sqrt{3})^{-\frac{N+1}{2}} \equiv-1(\bmod N) \equiv-1\left(\bmod q_{i}\right) \quad(i=1,2,3)
$$

evidently is that

$$
\frac{N+1}{2} \text { is an odd multiple of } \frac{q_{i}+1}{2} \quad(i=1,2,3) .
$$

Now, suppose that any two of the numbers $\left(q_{i}+1\right) / 8(i=1,2,3)$ have the same greatest common divisor $d$ and that

$$
\frac{q_{i}+1}{2}=\frac{48 n_{i}+7+1}{2}=4\left(6 n_{i}+1\right)=4 d p_{i} \quad(i=1,2,3) .
$$

We conclude that

$$
\frac{N+1}{2}=\frac{q_{1} q_{2} q_{3}+1}{2}=\frac{\left(8 d p_{1}-1\right)\left(8 d p_{2}-1\right)\left(8 d p_{3}-1\right)+1}{2}
$$

should be an odd multiple of $4 d p_{1} p_{2} p_{3}$, or, which is the same thing, that

$$
64 d^{3} p_{1} p_{2} p_{3}-8 d^{2}\left(p_{1} p_{2}+p_{1} p_{3}+p_{2} p_{3}\right)+d\left(p_{1}+p_{2}+p_{3}\right)
$$

should be an odd multiple of $d p_{1} p_{2} p_{3}$.

Since $d$ and the $p_{i}$ must be odd numbers, the number (12) is odd, and so this number is an odd multiple of $d p_{1} p_{2} p_{3}$ if or

$$
64 d^{3} p_{1} p_{2} p_{3}-8 d^{2}\left(p_{1} p_{2}+p_{1} p_{3}+\dot{p}_{2} p_{3}\right)+d\left(p_{1}+p_{2}+p_{3}\right) \equiv 0\left(\bmod d p_{1} p_{2} p_{3}\right)
$$

$$
8 d\left(p_{1} p_{2}+p_{1} p_{3}+p_{2} p_{3}\right) \equiv p_{1}+p_{2}+p_{3}\left(\bmod p_{1} p_{2} p_{3}\right) .
$$

Since $d p_{i}=6 n_{i}+1$, all the numbers $d$ and $p_{i}$ must be of one of the forms $6 s+1$ or $6 s-1$. Now, for the $p_{i}$ choose 3 numbers, relatively prime in pairs, and of one of the forms $6 s \pm 1$, e.g.

$$
p_{1}=1, p_{2}=7 \text { and } p_{3}=13 \text {. }
$$

Congruence (13) becomes

$$
8 d \cdot 111 \equiv 21(\bmod 91)
$$

with the extra condition

$$
d \equiv 1(\bmod 6) \text {. }
$$




\section{H. RIESEL, Prime numbers of two forms}

These two last congruences have the solutions

and we find

$$
d \equiv 301(\bmod 546)=546 x+301,
$$

and

$$
\begin{aligned}
& q_{1}=8 d p_{1}-1=8 d-1=4368 x+2407 \\
& q_{2}=8 d p_{2}-1=56 d-1=30576 x+16855
\end{aligned}
$$

$$
q_{3}=8 d p_{3}-1=104 d-1=56784 x+31303 .
$$

The only thing that remains is to determine an $x$ for which all $q_{i}$ are primes; the prime tables show that the values $x=37$ and $x=89 \mathrm{fit}$, and so we have:

$$
N_{1}=164023 \cdot 1148167 \cdot 2132311
$$

and

$$
N_{2}=391159 \cdot 2738119 \cdot 5085079
$$

are composed numbers for which congruence (6) holds.

In the same way we can construct an example to show that the inequality

$$
2^{2 n}-1 \geqq a
$$

in Theorem 2 could not simply be removed. We have found

$$
M=327823 \cdot 2294767 \cdot 4261711 \text {. }
$$

Numerical example. For the number $M=5 \cdot 2^{14}-1=81919$ the conditions of theorem 2 are fulfilled, and we find

$$
\begin{aligned}
& v_{1}=724, v_{2} \equiv 32660, v_{3} \equiv 8299, v_{4} \equiv 61439, v_{5} \equiv 5118, \\
& v_{6} \equiv 61761, v_{7} \equiv 26722, v_{8} \equiv 59278, v_{9} \equiv 47696, v_{10} \equiv 17784, \\
& v_{11} \equiv 63314, v_{12} \equiv 38248 \text { and } v_{13} \equiv 0, \text { all congruences taken (mod 81919). }
\end{aligned}
$$

The last congruence shows that the number 81919 is a prime.

Putting $a=2$, we find $u_{1}=27246964$ and $v_{1}=1956244$.

Putting $a=3$, we find $u_{1}=73621286644$ and $v_{1}=5285770564$.

Theorems 1 and 2 were used to find the prime character of some numbers $(6 a \pm 1) 2^{e}-1$. The calculations were made on the Swedish high speed electronic computer BESK for all values of $6 a \pm 1<56$ and $e \leqq 150$, except for $6 a \pm 1=5,7$ and 11 , where $e$ takes all values $\leqq 250$.

Those numbers which were found to be primes were tested once more by BESK. The result was that the numbers $N$ and $M$ were prime for the following values of $6 a \pm 1$ and $e$, and composite otherwise (the values of $e=1,2,3$ are taken from the prime tables): 
ARKIV För MATEMATIK. Bd 3 nr 20

\begin{tabular}{|c|l|}
\hline $6 a \pm 1$ & || \\
\hline 5 & $2,4,8,10,12,14,18,32,48,54,72,148,184,248$ \\
7 & $1,5,9,17,21,29,45,177$ \\
11 & $2,26,50,54,126,134,246$ \\
& \\
\cline { 2 - 2 } & \\
13 & $3,7,23$ \\
17 & $2,4,6,16,20,36,54,60,96,124,150$ \\
19 & $1,3,5,21,41,49,89,133,141$ \\
23 & $4,6,12,46,72$ \\
25 & $3,9,11,17,23,35,39,75,105,107$ \\
29 & $4,16,76,148$ \\
31 & $1,5,7,11,13,23,33,35,37,47,115$ \\
35 & $2,6,10,20,44,114,146$ \\
37 & 1 \\
41 & $2,10,14,18,50,114,122$ \\
43 & $7,31,67$ \\
47 & $4,14,70,78$ \\
49 & $1,5,7,9,13,15,29,33,39,55,81,95$ \\
53 & $2,6,8,42,50,62$ \\
55 & $1,3,5,7,15,33,41,57,69,75,77,131,133$ \\
\hline
\end{tabular}

BESK also verified that the known Mersenne numbers up to $2^{607}-1$ are primes.

\section{REFEREN GES}

1. D. H. LEHMER, Annals of Math. (2), 31, 446 (1930).

2. B. W. Brewer, Duke Math. Journ. 18, 757 (1951).

3. A. E. Western, Journ. of the London Math. Soc. 7, 130 (1932).

Tryckt den 7 maj 1955

Uppsala 1955. Almqvist \& Wiksells Boktryckeri AB 